\title{
A Rough Type-2 Fuzzy Clustering Algorithm for MR Image Segmentation
}

\author{
Shahin Ara Begum \\ O. Mema Devi
}

Department of Computer Science, Assam University, Silchar - 788011

\begin{abstract}
As medical images contain uncertainties, there are difficulties in classification of images into homogeneous regions. Fuzzy sets, rough sets and the combination of fuzzy and rough sets plays a prominent role in formalizing uncertainty, vagueness, and incompleteness in diagnosis. Development of hybrid approaches for the segmentation of the magnetic resonance imaging (MRI) with the ability of combining the merits of individual techniques is constantly increasing. The roughfuzzy $c$-means (RFCM) clustering algorithm is a hybrid approach combining the merits of fuzzy set and rough set in the $c$-means framework and is successfully applied to the MR image segmentation. The focus of this paper is on enhancing the computational capability of the fuzzy set based clustering algorithms and the hybrid variant. In this paper we propose an alternative optimal version of the hybrid variant based on type-2 membership function and rough set. We extend and generalized RFCM algorithm with type-2 membership function and call it rough type-2 fuzzy $c$-means (RT2FCM) algorithm. The RT2FCM algorithm is a generalization of the RFCM algorithm which extends the membership value of each pattern to the type- 2 membership functions and is applied to the segmentation of MR images. Experimentation is done using the brain MR images and the results show better detection of abnormal tissues by RT2FCM in contrast to the FCM, T2FCM (Type-2 FCM) and RFCM clustering algorithms.
\end{abstract}

\section{Keywords}

Fuzzy $c$-means, Rough-fuzzy $c$-means, MR imaging, image segmentation.

\section{INTRODUCTION}

The concept of fuzzy set which was first introduced by Zadeh [1] and rough set which was first introduced by Pawlak [2] theories are the generalization of the crisp set theory for formalizing the incomplete, uncertain and vague information and are useful in the field of medical diagnosis as medical knowledge consists of medical descriptions and assertions that are incomplete and uncertain. Fuzzy sets and rough sets are incorporated in the $c$-means framework to develop the FCM (fuzzy c-means) and RFCM (rough fuzzy $c$-means) clustering algorithms. The FCM clustering algorithm was proposed by Bezdek [3] by extending the membership logic in the hard $c$ means (crisp clustering) algorithm and is the best known and powerful methods used in cluster analysis. In crisp clustering, data set is characterized by crisp set where elements i.e. the data points definitely belong to the set characterizing the cluster. Fuzzy clustering allows each feature vector to belong to more than one cluster with different membership degrees in the interval $[0,1]$ and there are vague or fuzzy boundaries between the clusters. The RFCM clustering algorithm is a hybrid approach where the fuzzy sets and rough sets are incorporated in the $c$-means framework to develop stronger models of uncertainty and is successfully applied to MR image segmentation [4]. This algorithm adds the concept of fuzzy membership of fuzzy sets, lower and upper approximation of rough sets into $c$-means algorithm. In fuzzy clustering, each object is characterized by the partial membership whereas in rough clustering objects are characterized using the concept of a boundary region with two approximations-lower and upper approximation [5] [6]. The lower approximation of a rough cluster contains objects that only belong to that cluster and is a subset of the upper approximation of the same cluster. The upper approximation of a rough cluster contains objects in the cluster which are also members of other cluster. The advantage of using rough sets is that, unlike other techniques, rough set theory does not require any prior information about the data such as membership function in fuzzy set theory. The fuzzy set of type-2 first introduced by Zadeh [7] is a generalization of the concept of ordinary fuzzy set theory where each object is characterized by a fuzzy membership function (or fuzzy grade) of which is a fuzzy set in the unit interval $[0,1]$ rather than a point in the interval $[0,1]$.

Modern neurosurgery takes the advantages of MR imaging of the patient before the diagnostic procedure. The principle of MR imaging was discovered in the late 1940s and has become more powerful and useful because of its ability to measure spatial distribution between the anatomical structures of biological tissue as compared to other medical imaging modalities [8] [9]. MRI is a medical imaging technique that uses nuclear magnetic resonance of protons to produce proton density images. The image pixel value can be considered as subsets of parameters including the time constants characterization T1 (magnetization vector along with longitudinal axis) and T2 (transverse component) and proton density (that has distinct value). Another MR imaging scan is diffusion MRI and it measures the diffusion of water molecules in biological tissues. By changing the effect of these meters, MR images can differentiate the structures obtained from the same anatomical positions. In diagnosis and prognosis using MR imaging, segmentation is often required to extract the interested and meaningful structure on the image and is considered as an important basic operation for meaningful analysis and interpretation of acquired images. Segmentation subdivides an image into its constituent regions or objects that have similar properties to produce anatomical structures [10] and the quality of any image interpretation depends on it. MR image segmentation using pattern recognition methods plays a vital role for early diagnosis by detecting the abnormal changes in tissues and organs. As the image segmentation is fundamentally a clustering problems, the techniques based on fuzzy clustering plays a vital role in MR image to analyze the patient's data (determine the exact location of an organ) and was introduced by Bezdek and for 
the first time [11] applied this in brain tissue to give visual representation of the original data. Any improvement in segmentation methods can lead to important impacts on MR image processing technique.

In this paper, we extend the RFCM with type-2 fuzzy set where the membership value of each pattern in the data is extended as type-2 fuzzy memberships. The proposed algorithm RT2FCM is applied to the brain MR image segmentation and the outcome of the RT2FCM algorithm is compared with the outcome of the FCM, T2FCM and the RFCM algorithm according to the value of the standard image quality indices like mean squared error (MSE) and image quality index (IQI). From the experimental results, it is found that the RT2FCM algorithm has better performance and accuracy as compared to the existing algorithms.

The remainder of this paper is structured as follows: Section 2 provides the outline of the existing clustering algorithms viz. FCM, T2FCM and RFCM. Section 3 presents the enhanced RT2FCM algorithm and in section 4 we apply the algorithms to the MR image segmentation and present the results. Finally section 5 concludes the paper.

\section{BACKGROUND}

This section presents the outline of the FCM, T2FCM and the RFCM algorithm used in the present study.

\subsection{Fuzzy $c$-means Algorithm}

FCM is a fuzzy version of the existing $c$-means algorithm and is based on a fuzzy extension of the least-square error criterion. The advantage of FCM is that it assigns each pattern to each cluster with some degree of membership (i.e. fuzzy clustering). This is more suitable for real applications where there are some overlaps between the clusters in the data set. Thus, FCM is a method of clustering which allows one piece of data to belong to two or more clusters. This method is frequently used in pattern recognition in medical diagnosis and is described in brief next.

Let $X=\left\{x_{1}, x_{2} \ldots x_{n}\right\}$ be a data set and let $c$ be a positive integer greater than one. A fuzzy pseudopartition or fuzzy $c$-partition of $X$ is a family of fuzzy subsets of $X$, denoted by $P=\left\{\mu_{1}\right.$, $\left.\mu_{2} \ldots \mu_{c}\right\}$, which satisfies

$\sum_{i=1}^{c} \mu_{i}\left(x_{j}\right)=1, \quad \forall x_{j} \in X$

The FCM minimizes the following objective function:

$J_{F C M}=\sum_{i=1}^{c} \sum_{j=1}^{n} \mu_{i j}^{m}\left\|x_{j}-v_{i}\right\|^{2}$

The parameter $m$ is a weight that determines the degree to which partial members of a cluster affect the clustering result. The FCM clustering algorithm is iteration through the necessary conditions for minimizing $J_{F C M}$ with the following update equations:

$v_{i}=\frac{\sum_{j=1}^{n} \mu_{i j}{ }^{m} x_{j}}{\sum_{j=1}^{n} \mu_{i j}{ }^{m}}, \quad i=1,2, \ldots, c$

and

$$
\begin{gathered}
\mu_{i j}=\left(\sum_{k=1}^{c} \frac{\left\|x_{j}-v_{i}\right\|^{2 /(m-1)}}{\left\|x_{j}-v_{k}\right\|^{2 /(m-1)}}\right)^{-1}, \\
i=1,2, \ldots, c ; j=1,2, \ldots, n
\end{gathered}
$$

The algorithm is based on the assumptions that the desired number of clusters $c$ is given and in addition, a particular distance, a real number $m \in(1, \infty)$, and a small positive number $\varepsilon$, serving as the stopping criterion, are chosen. The process stops when the centroid stabilizes. That is, the centroids of the previous iteration are identical to those generated in the current iteration. The FCM algorithm is as follows [3] [12] [13]:

S1: Fix $m>1$ and $2 \leq c \leq n-1$ and give $c$ initial cluster centers $v_{i}$.

REPEAT

$\mathrm{S} 2$ : Compute $\mu_{i j}$ with $v_{i}$ by Eq. (3).

S3: Compute the objective function $J_{F C M}$ by Eq. (1).

S4: Update $v_{i}$ with $\mu_{i j}$ by Eq. (2).

UNTIL (cluster centers stabilize).

The number of iteration taken by the algorithm depends on the position of the initial cluster prototypes. For random initialization of cluster prototype the algorithm converge to the desired results in less number of iterations if the initial cluster prototypes lies near the actual ones. Otherwise, the algorithm takes more iteration to find the actual prototypes. Thus, FCM clustering algorithm is sensitive to position of initial cluster prototypes. So, computational complexity is higher in FCM for a bad prototype initialization. Developments of the variations of the FCM algorithm are constantly increasing to perform well with medical data sets.

\subsection{Type-2 Fuzzy $c$-means Algorithm}

The type-2 fuzzy set is a generalization of the concept of ordinary fuzzy set theory and much research is continuing to be done [14] [15] [16] [17] [18]. Each membership functions in type-1 fuzzy set are always a crisp set into real numbers in $[0,1]$ whereas the membership functions of type-2 fuzzy set are ordinary fuzzy sets i.e., 'fuzzy-fuzzy set'. Thus the type-2 fuzzy set can be defined as a generalized fuzzy set by allowing their intervals to be fuzzy i.e., fuzziness of a fuzzy set. The advantage of type- 2 fuzzy logic is that it takes one more step toward the use of computers to represent the human perception.

The membership values for the type- 2 fuzzy set are obtained as:

$a_{i j}=\mu_{i j}-\frac{1-\mu_{i j}}{2}$

Where $\mu_{i j}$ and $a_{i j}$ are the primary membership (i.e., the membership of type- 1 fuzzy set) and the type- 2 membership respectively. The type- 2 membership function area can be considered as the uncertainty of type-1 membership contribution when the center is updated. The cluster centers are updated using the following equation.

$v_{i}=\frac{\sum_{j=1}^{n} a_{i j}{ }^{m} x_{j}}{\sum_{j=1}^{n} a_{i j}{ }^{m}}, \quad i=1,2, \ldots, c$

During the cluster center updates, the contribution of a pattern that has low memberships to a given cluster is relatively smaller when using type-2 memberships and the memberships may represent better typicality. Cluster centers that are estimated by type- 2 memberships tend to have more desirable locations than cluster centers obtained by type-1 FCM method in the presence of noise.

The T2FCM objective function is defined as: 


$$
J_{T 2 F C M}=\sum_{i=1}^{c} \sum_{j=1}^{n} a_{i j}^{m}\left\|x_{j}-v_{i}\right\|^{2}
$$

The type-2 FCM algorithm proceeds as in FCM, along with the incorporation of type- 2 membership. Thus, we have the T2FCM algorithm as follows:

S1: Fix $m>1$ and $2 \leq c \leq n-1$ and give $c$ initial cluster centers $v_{i}$

\section{REPEAT}

S2: Compute $\mu_{i j}$ with $v_{i}$ by Eq. (3).

S3: Compute $a_{i j}$ with $\mu_{i j}$ by Eq. (4).

S4: Compute the objective function $J_{T 2 F C M}$ by Eq. (6).

S5: Update $v_{i}$ with $a_{i j}$ by Eq. (5).

UNTIL (cluster centers stabilized).

\subsection{Rough-Fuzzy $c$-means algorithm}

In the computational intelligence community, hybrid approaches have attracted considerable attention and there has been increased interest in development of hybrid approaches for MR image segmentation. The RFCM algorithm is one such hybrid approach which is the integration of rough set and fuzzy set in $c$-means framework. While the membership of fuzzy sets enables efficient handling of overlapping partitions, the rough sets deal with uncertainty, vagueness and incompleteness in class definition. The RFCM [4] partitions a set of $n$ objects into $c$ clusters by minimizing the objective function:

$$
\begin{aligned}
J_{R F} & = \begin{cases}w \times A_{1}+\widetilde{w} \times B_{1} \text { if } \underline{A}\left(\beta_{i}\right) \neq \emptyset, B\left(\beta_{i}\right) \neq \varnothing \\
A_{1} & \text { if } \underline{A}\left(\beta_{i}\right) \neq \emptyset, B\left(\beta_{i}\right)=\emptyset \\
B_{1} & \text { if } \underline{A}\left(\beta_{i}\right)=\emptyset, B\left(\beta_{i}\right) \neq \emptyset\end{cases} \\
A_{1} & =\sum_{i=1}^{c} \sum_{x_{j} \in A\left(\beta_{i}\right)}\left\|x_{j}-v_{i}\right\|^{2} \\
B_{1} & =\sum_{i=1}^{c} \sum_{x_{j} \in B\left(\beta_{i}\right)}\left(\mu_{i j}\right)^{m}\left\|x_{j}-v_{i}\right\|^{2}
\end{aligned}
$$

$v_{i}$ represents the centroid of the $i^{\text {th }}$ cluster $\beta_{i}, \underline{A}\left(\beta_{i}\right)$ and $\bar{A}\left(\beta_{i}\right)$ be the lower and upper approximations of cluster $\beta_{i}$ and $B\left(\beta_{i}\right)=\left(\bar{A}\left(\beta_{i}\right)-\underline{A}\left(\beta_{i}\right)\right)$ denote the boundary region of cluster $\beta_{i}$. The parameter $w$ and $\widetilde{w}$ correspond to the relative importance of lower bound and boundary region and $w+$ $\widetilde{w}=1$. Also $\mu_{i j}$ has the same meaning of membership as that in FCM.

The new centroid is calculated based on the weighting average of the crisp lower approximation and fuzzy boundary. Computation of the centroid is modified to include the effects of both fuzzy memberships and lower and upper bounds. The modified centroid calculation for the RFCM is obtained by solving Eq. (6) with respect to $v_{i}$

$$
\begin{aligned}
& v_{i}^{R F}= \begin{cases}w \times C_{1}+\widetilde{w} \times D_{1} \text { if } \underline{A}\left(\beta_{i}\right) \neq \emptyset, B\left(\beta_{i}\right) \neq \emptyset \\
C_{1} & \text { if } \underline{A}\left(\beta_{i}\right) \neq \emptyset, B\left(\beta_{i}\right)=\emptyset \\
D_{1} & \text { if } \underline{A}\left(\beta_{i}\right)=\varnothing, B\left(\beta_{i}\right) \neq \emptyset\end{cases} \\
& C_{1}=\frac{1}{\left|\underline{A}\left(\beta_{i}\right)\right|} \sum_{x_{j} \in \underline{A}\left(\beta_{i}\right)} x_{j}
\end{aligned}
$$

where $\left|\underline{A}\left(\beta_{i}\right)\right|$ represents the cardinality of $\underline{A}\left(\beta_{i}\right)$ and

$$
D_{1}=\frac{1}{n_{i}} \sum_{x_{j} \in B\left(\beta_{i}\right)}\left(\mu_{i j}\right)^{m} x_{j} \text { where } n_{i}=\sum_{x_{j} \in B\left(\beta_{i}\right)}\left(\mu_{i j}\right)^{m}
$$

Thus, the cluster prototypes (centroids) depend on the parameters, $w$ and $\widetilde{w}$ and fuzzifier $m$ rule their relative influence. The correlated influence of these parameters and fuzzifier, makes it difficult to determine their optimal values. Since the objects lying in lower approximation definitely belong to a cluster, they are assigned a higher weight $w$ compared to $\widetilde{w}$ of the objects lying in boundary region and $0<\widetilde{w}<w<1$.

The RFCM algorithm starts by randomly choosing $c$ objects as the centroids of the $c$ clusters. The fuzzy memberships of all objects are calculated using Eq. (3).

Let $\mu_{i}=\left(\mu_{i 1}, \mu_{i 2}, \ldots, \mu_{i j}, \ldots, \mu_{i n}\right)$ represent the fuzzy cluster $\left(\beta_{i}\right)$ associated with the centroid $v_{i}$. After computing $\mu_{i j}$ for $c$ clusters and $n$ objects, the values of $\mu_{i j}$ for each object $x_{j}$ are sorted and the difference of two highest memberships of $x_{j}$ is compared with a threshold value $\delta$. Let $\mu_{i j}$ and $\mu_{k j}$ be the highest and second highest memberships of $x_{j}$. If $\left(\mu_{i j}-\right.$ $\left.\mu_{k j}\right)>\delta$, then $x_{j} \in \underline{A}\left(\beta_{i}\right)$ as well as $x_{j} \in \bar{A}\left(\beta_{i}\right)$, otherwise $x_{j} \in \bar{A}\left(\beta_{i}\right)$ and $x_{j} \in \overline{\bar{A}}\left(\beta_{k}\right)$. After assigning each object in lower approximations or boundary regions of different clusters based on $\delta$, memberships of $\mu_{i j}$ of the objects are modified. The values of $\mu_{i j}$ are set to 1 for the objects in lower approximations, while those in boundary regions are remain unchanged. The new centroids of the clusters are calculated as per Eq. (8).Thus, the RFCM algorithm is summarized as follows:

S1: Assign the initial centroids $v_{i}$, choose values for fuzzifier REPEAT $m$, and thresholds $\varepsilon$. Set iteration counter $t=1$

S2: Compute by $\mu_{i j}$ Eq. (3).

S3: If $\mu_{i j}$ and $\mu_{k j}$ be the two highest memberships of $x_{j}$ and $\left(\mu_{i j^{-}} \mu_{k j}\right) \leq \delta$, then $x_{j} \in \bar{A}\left(\beta_{i}\right)$ and $x_{j} \in \bar{A}\left(\beta_{k}\right)$. Furthermore, $x_{j}$ is not part of any lower bound.

S4: Otherwise, $x_{j} \in \underline{A}\left(\beta_{i}\right)$. In addition, by properties of rough sets, $x_{j} \in \bar{A}\left(\beta_{i}\right)$.

S5: Modify $\mu_{i j}$ considering lower and boundary regions for $c$ clusters and $n$ objects.

S6: Compute the objective function $J_{R F}$ by Eq. (7).

S7: Compute new centroids using Eq. (8).

UNTIL (cluster centers stabilized).

The performance of the RFCM algorithm depends on the value of $\delta$, which represents the size of the granules of rough fuzzy clustering. For implementation of this algorithm, we have applied the following definition for $\delta$ i.e., the average difference of two highest memberships of all the objects in the data set:

$\delta=\frac{1}{n} \sum_{j=1}^{n}\left(\mu_{i j}-\mu_{k j}\right)$

\section{THE ROUGH TYPE-2 FUZZY $C$ - MEANS (RT2FCM) ALGORITHM}

This section presents the proposed RT2FCM wherein we extend the membership function of the FCM in order to increase the performance of the rough fuzzy based clustering algorithm (RFCM) in terms of the detection of the abnormal tissues of the MR image. To improve the performance of the RFCM algorithm we extend the membership function with the 
type-2 fuzzy membership function. In this algorithm, the type2 membership function is added to the RFCM algorithm. The membership value for type-2 is calculated by using Eq. (4) and using the membership value, the cluster centers are updated. The objective function is obtained with the type- 2 membership values as:

$J_{R T 2 F}= \begin{cases}w \times A_{1}+\widetilde{w} \times B_{1} & \text { if } \underline{A}\left(\beta_{i}\right) \neq \emptyset, B\left(\beta_{i}\right) \neq \emptyset \\ A_{1} & \text { if } \underline{A}\left(\beta_{i}\right) \neq \emptyset, B\left(\beta_{i}\right)=\varnothing \\ B_{1} & \text { if } \underline{A}\left(\beta_{i}\right)=\varnothing, B\left(\beta_{i}\right) \neq \varnothing\end{cases}$

$A_{1}=\sum_{i=1}^{c} \sum_{x_{j} \in \underline{A}\left(\beta_{i}\right)}\left\|x_{j}-v_{i}\right\|^{2}$,

$B_{1}=\quad \sum_{i=1}^{c} \sum_{x_{j} \in B\left(\beta_{i}\right)}\left(a_{i j}\right)^{m}\left\|x_{j}-v_{i}\right\|^{2}$

$v_{i}$ represents the centroid of the $i^{\text {th }}$ cluster $\beta_{i}$, the $\underline{A}\left(\beta_{i}\right)$, $\bar{A}\left(\beta_{i}\right)$ and the parameter $w$ and $\widetilde{w}$ has the same meaning as in type-1 RFCM. Also $a_{i j}$ has the same meaning of membership as that in type-2 FCM.

The value for $\delta$ is obtained as:

$\delta=\frac{1}{n} \sum_{j=1}^{n}\left(a_{i j}-a_{k j}\right)$

Where $a_{i j}$ and $a_{k j}$ are the first and second highest membership values of type-2 fuzzy set respectively.After computing $a_{i j}$ for $c$ clusters and $n$ objects, the values of $a_{i j}$ for each object $x_{j}$ are sorted and the difference of two highest memberships of $x_{j}$ is compared with a threshold value $\delta$. If $\left(a_{i j}-a_{k j}\right)>\delta$, then $x_{j} \in \underline{A}\left(\beta_{i}\right)$ as well as $x_{j} \in \bar{A}\left(\beta_{i}\right)$, otherwise $x_{j} \in \bar{A}\left(\beta_{i}\right)$ and $x_{j} \in \bar{A}\left(\beta_{k}\right)$. After assigning each object in lower approximations or boundary regions of different clusters based on $\delta$, memberships of $a_{i j}$ of the objects are modified. The values of $a_{i j}$ are set to 1 for the objects in lower approximations, while those in boundary regions are remain unchanged. The new centroids of the clusters are calculated as per Eq. (12). Thus the RT2FCM algorithm is as follows:

S1: Assign the initial centroids $v_{i}$, choose values for fuzzifier REPEAT $m$, and thresholds $\varepsilon$. Set iteration counter $t=1$

S2: Compute $\mu_{i j}$ with $v_{i}$ by Eq. (3).

S3: Compute $a_{i j}$ with $\mu_{i j}$ by Eq. (4).

S4: If $a_{i j}$ and $a_{k j}$ be the two highest memberships of $x_{j}$ and $\left(a_{i j^{-}}\right.$ $\left.a_{k j}\right) \leq \delta$, then $x_{j} \in \bar{A}\left(\beta_{i}\right)$ and $x_{j} \in \bar{A}\left(\beta_{k}\right)$. Furthermore, $x_{j}$ is not part of any lower bound.

S5: Otherwise, $x_{j} \in \underline{A}\left(\beta_{i}\right)$. In addition, by properties of rough sets, $x_{j} \in \bar{A}\left(\beta_{i}\right)$.

S6: Modify $a_{i j}$ considering lower and boundary regions for $c$ clusters and $n$ objects.

S7: Compute the objective function $J_{T 2 R F}$.

S8: Compute new centroids as:

$v_{i}^{R F}= \begin{cases}w \times C_{1}+\widetilde{w} \times D_{1} \text { if } \underline{A}\left(\beta_{i}\right) \neq \emptyset, B\left(\beta_{i}\right) \neq \varnothing \\ C_{1} & \text { if } \underline{A}\left(\beta_{i}\right) \neq \emptyset, B\left(\beta_{i}\right)=\emptyset \\ D_{1} & \text { if } \underline{A}\left(\beta_{i}\right)=\emptyset, B\left(\beta_{i}\right) \neq \emptyset\end{cases}$

$C_{1}=\frac{1}{\left|\underline{A}\left(\beta_{i}\right)\right|} \sum_{x_{j} \in \underline{A}\left(\beta_{i}\right)} x_{j}$
Where $\left|\underline{A}\left(\beta_{i}\right)\right|$ represents the cardinality of $\underline{A}\left(\beta_{i}\right)$ and

$D_{1}=\frac{1}{n_{i}} \sum_{x_{j} \in B\left(\beta_{i}\right)}\left(a_{i j}\right)^{m} x_{j}$ Where $n_{i}=\sum_{x_{j} \in B\left(\beta_{i}\right)}\left(a_{i j}\right)^{m}$

UNTIL (cluster centers stabilize).

The performance of the RT2FCM algorithm also depends on the value of $\delta$, which represents the size of the granules of type- 2 rough fuzzy clustering.

\section{RESULTS AND DISCUSSION}

In this section the scope is to compare the developed RT2FCM algorithm with different algorithms including FCM, T2FCM and the RFCM algorithm.

\subsection{Data Sets}

For the performance analysis of the algorithms we have conducted experiments on several MR images [19]. The first data set is the Mets brain MR images from a patient with brain metastatic neoplasm. These images are the original image for MRI of T1, T2, proton density (PD) and diffusion weighted respectively. All the images are in JPEG format and it is of $737 \times 965,649 \times 867,753 \times 985$ and $667 \times 879$ bit resolution grayscale version of the original images respectively. The second data set is the Anaplastic astrocytoma, also known as a grade 3 astrocytoma. All the images are in JPEG format and it is of $751 \times 899,687 \times 873,773 \times 917$ and $641 \times 817$ bit resolution grayscale version of the original images respectively.

\subsection{Performance measure}

To evaluate the performance of the segmentation results of the algorithms, the Mean Squared Error (MSE) and the image quality index (IQI) has been used.

\subsubsection{Mean Squared Error (MSE)}

The MSE often refer to the error signal which is the difference between the original and the test image (segmented image) signals. If one of the signals is an original signal of acceptable quality, and the other is a segmented image of it whose quality is being evaluated, then MSE may also be regarded as a measure of image quality. The MSE is defined as [20]:

$\operatorname{MSE}(x, y)=\frac{1}{N} \sum_{i=1}^{N}\left(x_{i}-y_{i}\right)$

Where $x=\left\{x_{i} \mid i=1,2, \ldots, N\right\}$, and $y=\left\{y_{i} \mid i=1,2, \ldots, N\right\}$ are the original and the test image (segmented image) signals, respectively and $N$ is the total number of patterns/pixels. MSE is used not only to evaluate, but also to optimize a large variety of algorithms for images such as medical pattern recognition. Minimizing MSE is a key criterion in selection estimators.

\subsubsection{Image Quality Index (IQI)}

The new principle behind the image quality metrics is: "The main function of the human eyes is to extract structural information from the viewing field, and the human visual system is highly adapted for this purpose. Therefore, a measurement of structural distortion should be a good approximation of perceived image distortion." Large errors do 
not always result in large structural distortions. The key point of the new philosophy is the switch from error measurement to structural distortion measurement. IQI is a relatively new universal objective image quality index, which is easy to calculate and applicable to various image processing applications. The image quality index (IQI) is defined as [21]:

$$
Q=\frac{4 \sigma_{x y} \overline{x y}}{\left(\sigma_{x}^{2}+\sigma_{y}^{2}\right)\left[(\bar{x})^{2}+(\bar{y})^{2}\right]}
$$

Where, $x=\left\{x_{i} \mid i=1,2, \ldots, N\right\}$, and $y=\left\{y_{i} \mid i=1,2, \ldots, N\right\}$ are the original and the test image (segmented image) signals, respectively,

$$
\begin{aligned}
& N=\text { total number of patterns/pixels, } \\
& \bar{x}=\frac{1}{N} \sum_{i=1}^{N} x_{i} \quad, \quad \bar{y}=\frac{1}{N} \sum_{i=1}^{N} y_{i} \\
& \sigma_{x}{ }^{2}=\frac{1}{N-1} \sum_{i=1}^{N}\left(x_{i}-\bar{x}\right)^{2}, \quad \sigma_{y}^{2}=\frac{1}{N-1} \sum_{i=1}^{N}\left(y_{i}-\bar{y}\right)^{2} \\
& \sigma_{x y}=\frac{1}{N-1} \sum_{i=1}^{N}\left(x_{i}-\bar{x}\right)\left(y_{i}-\bar{y}\right)
\end{aligned}
$$

Maximizing IQI is a key criterion in selection estimators. The best value 1 is achieved if and only if $y_{i}=x_{i}$ for all $i=1,2 \ldots$, $N$. The lowest value of -1 occurs when $y_{i}=2 \bar{x}-x_{i}$ for all $i=$ $1,2 \ldots, N$. From the literature it is found that MSE is more sensitive to the energy of errors instead of structural distortions as compared to IQI.

\subsection{Experimental Results}

The algorithms viz., FCM, T2FCM, RFCM and the developed RT2FCM clustering algorithms are implemented in MATLAB version 7.3 environments and performance analysis of the segmentation results are carried out on MR images. In order to examine the performance and accuracy of segmentation of the proposed algorithm (RT2FCM) and other existing clustering algorithms, the following criteria are used: (i) a random initialization to set as cluster prototypes, (ii) set $k=2$, $m=2, \varepsilon=0.01$ and $w=0.95$ for RFCM and RT2FCMand (iii) for each setup, the algorithms are run 10 times and the best case according to the value of the MSE and IQI indices are chosen. The figures 1(a-e), 2(a-e),3(a-e) and 4(a-e) shows the comparison of FCM, T2FCM, RFCM and RT2FCM segmented images for the Metsbrain MR images of T1, T2, PD and diffusion weight image respectively. The segmented results of the algorithms for Anaplastic astro MRI images for $\mathrm{T} 1, \mathrm{~T} 2$, PD and diffusion weight images are shown in figures $5(a-e), 6(a-d), 7(a-d)$ and $8(a-d)$ respectively. The quality of the segmented results for each algorithm is compared in the table 1(a) according to the values of the MSE and IQI. Further the computational time to complete the key operations for the algorithms is also compared in the table 1 (b). It is seen from the segmented results in the figures and table 1(a) that the RT2FCM segmentation has better detection of abnormal tissues according to the values of MSE and IQI indices as compared to the existing algorithms i.e., RFCM, T2FCM and FCM segmentation. In table 1(a) the entries in bold represent the optimal values. Further as compared to the FCM algorithm, the T2FCM and the RFCM algorithms have better performance according to the values of MSE and IQI indices. However, from table 1(b) it is seen that the computational time in terms of the CPU time to calculate the RFCM and the RT2FCM algorithm is larger than the FCM and T2FCM. It is also noted that with some data sets RT2FCM requires more CPU time than RFCM but with certain other data sets RFCM requires more CPU time than RT2FCM. In the same way T2FCM requires more CPU time than FCM with some data sets.

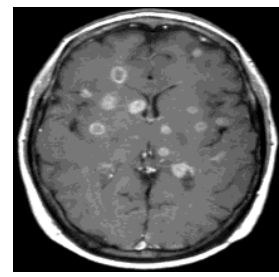

(a)

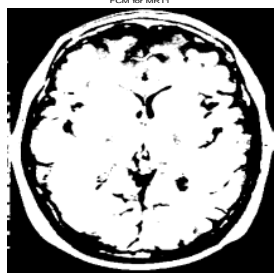

(b)

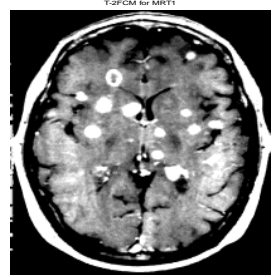

(c)

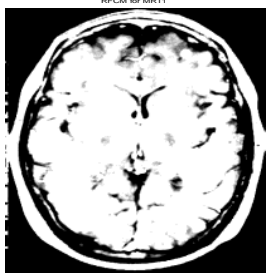

(d)

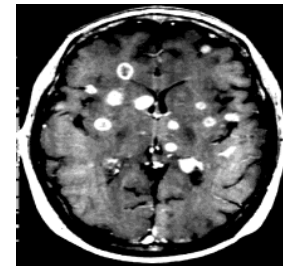

(e)

Figure 1: Segmentation results for the Metsbrain MR T1 data set (a) Original image (b) FCM segmentation (c) T2FCM segmentation (d) RFCM segmentation and (e) RT2FCM segmentation

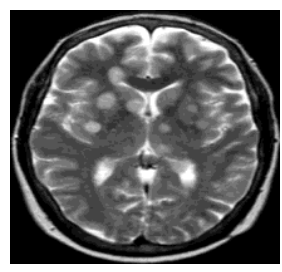

(a)

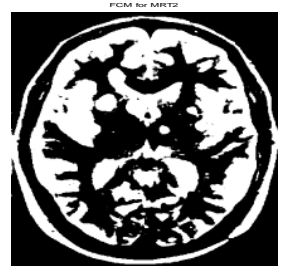

(b)

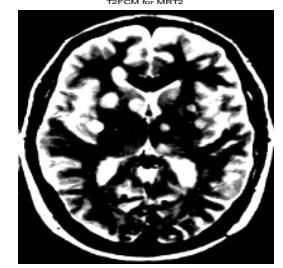

(c)

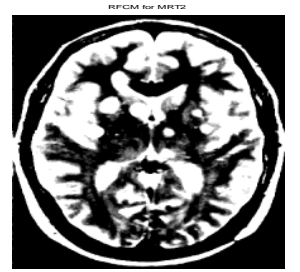

(d)

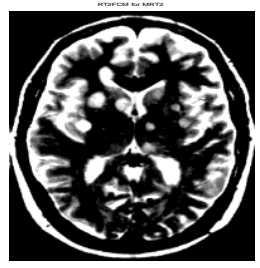

(e)

Figure 2: Segmentation results for the Mets brain MR T2 data set (a) Original image (b) FCM segmentation (c) T2FCM segmentation (d) RFCM segmentation and (e) RT2FCM segmentation 


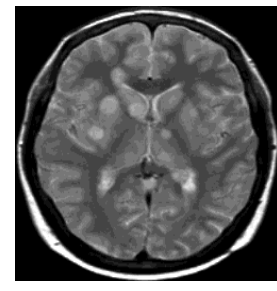

(a)

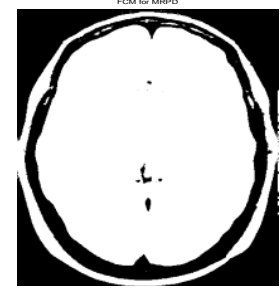

(b)

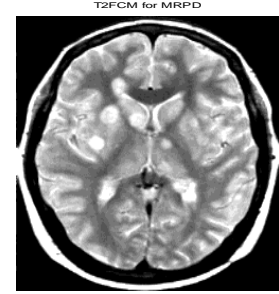

(c)

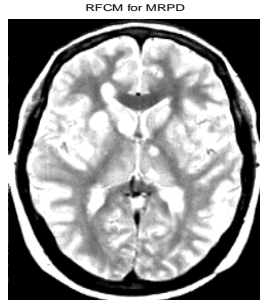

(d)

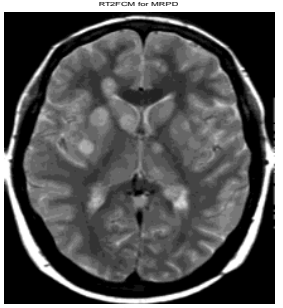

(e)

Figure 3: Segmentation results for the Metsbrain MR PD data set (a) Original image (b) FCM segmentation (c) T2FCM segmentation (d) RFCM segmentation and (e) RT2FCM segmentation

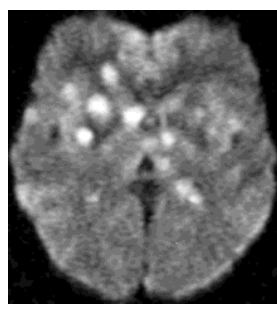

(a)

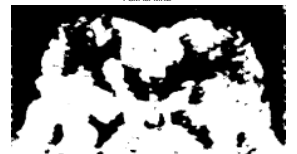

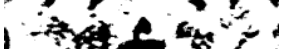

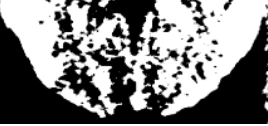

(b)
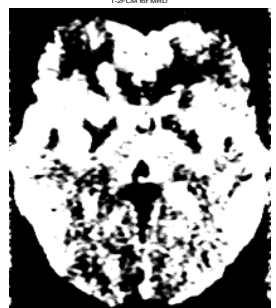

(c)
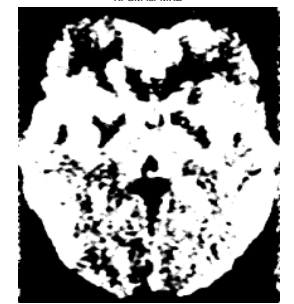

(d)

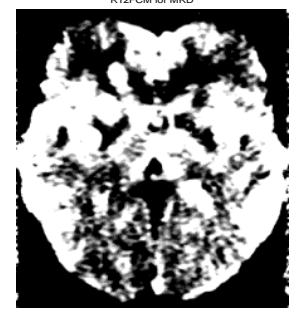

(e)

Figure 4: Segmentation results for the Metsbrain MR D data set (a) Original image (b) FCM segmentation (c) T2FCM segmentation (d) RFCM segmentation and (e) RT2FCM segmentation

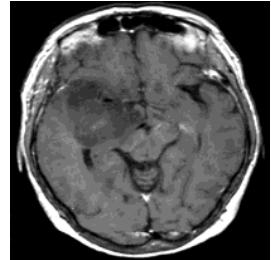

(a)

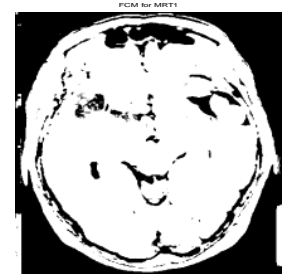

(b)

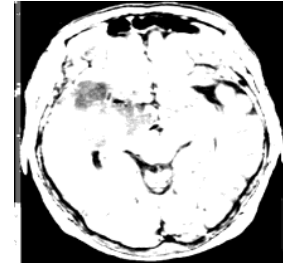

(c)

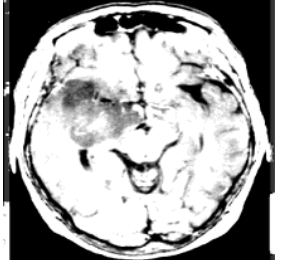

(d)

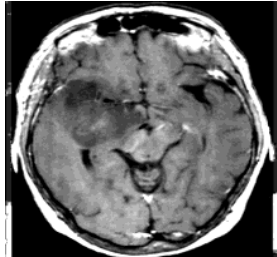

(e)

Figure 5: Segmentation results for the AnaplasticAstro MR T1 data (a) Original image (b) FCM segmentation (c) T2FCM segmentation (d) RFCM segmentation and (e) RT2FCM segmentation

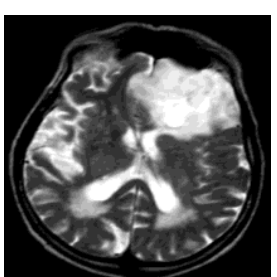

(a)

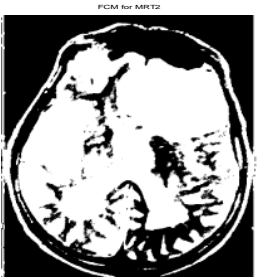

(b)

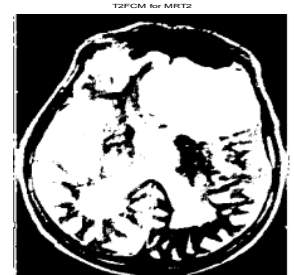

(c)

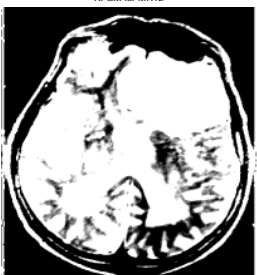

(d)

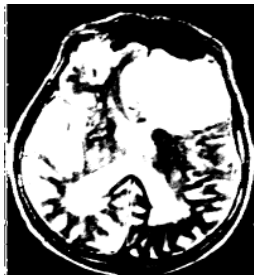

(e)

Figure 6: Segmentation results for the AnaplasticAstro MR T2 data (a) Original image (b) FCM segmentation (c) T2FCM segmentation (d) RFCM segmentation and (e) RT2FCM segmentation

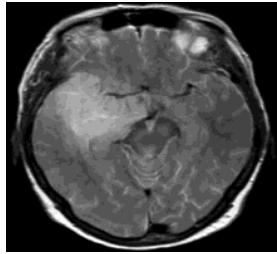

(a)

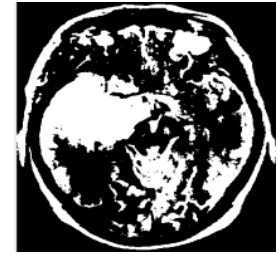

(b)

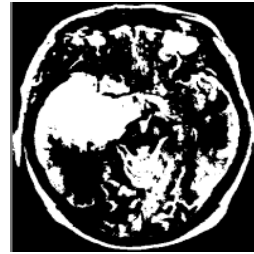

(c)

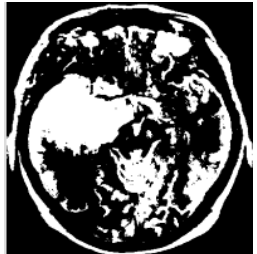

(d)

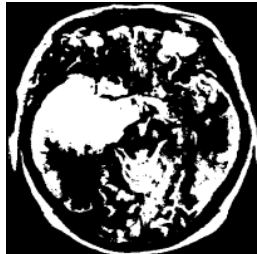

(e)

Figure 7: Segmentation results for the AnaplasticAstro MR PD data (a) Original image (b) FCM segmentation (c) T2FCM segmentation (d) RFCM segmentation and (e) RT2FCM segmentation 


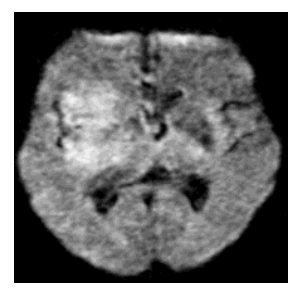

(a)

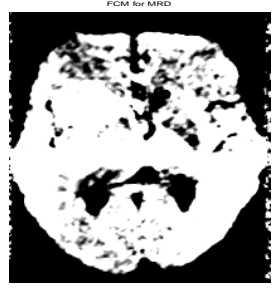

(b)

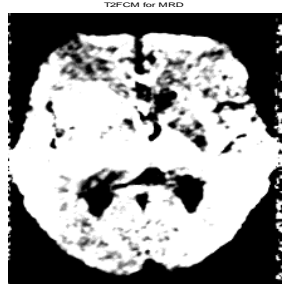

(c )

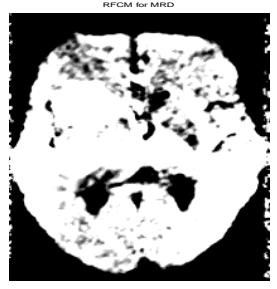

(d)

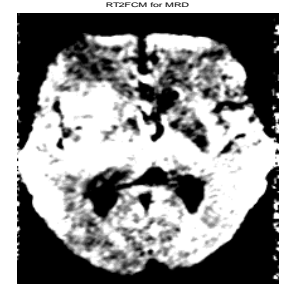

(e)

Figure 8: Segmentation results for the AnaplasticAstro MR D data (a) Original image (b) FCM segmentation (c) T2FCM segmentation (d) RFCM segmentation and (e) RT2FCM segmentation

Table 1(a): Performance of the FCM, T2FCM, RFCM and the RT2FCM algorithms for MR image segmentation (IQ and MSE values are the best case values)

\begin{tabular}{|c|c|c|c|c|c|c|c|c|c|}
\hline \multicolumn{2}{|c|}{ Data Set } & \multicolumn{2}{|c|}{ FCM } & \multicolumn{2}{|c|}{ T2FCM } & \multicolumn{2}{|c|}{ RFCM } & \multicolumn{2}{|c|}{ RT2FCM } \\
\hline & & IQ & MSE & IQ & MSE & IQ & MSE & IQ & MSE \\
\hline \multirow{4}{*}{ 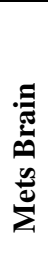 } & $\mathrm{T} 1$ & 0.5584 & 0.1680 & 0.8016 & 0.0385 & 0.5879 & 0.1568 & 0.8300 & 0.0285 \\
\hline & $\mathrm{T} 2$ & 0.6208 & 0.1091 & 0.7198 & 0.0633 & 0.6905 & 0.0805 & 0.7436 & 0.0537 \\
\hline & PD & 0.4759 & 0.2334 & 0.8033 & 0.0422 & 0.6772 & 0.0948 & 0.9521 & 0.0053 \\
\hline & D & 0.4848 & 0.1684 & 0.5340 & 0.1409 & 0.5137 & 0.1591 & 0.5776 & 0.1076 \\
\hline \multirow{4}{*}{ 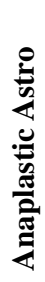 } & $\mathrm{T} 1$ & 0.4938 & 0.2258 & 0.5200 & 0.2132 & 0.5825 & 0.1603 & 0.8818 & 0.0204 \\
\hline & $\mathrm{T} 2$ & 0.5187 & 0.1815 & 0.5208 & 0.1791 & 0.5273 & 0.1785 & 0.5833 & 0.1412 \\
\hline & PD & 0.4884 & 0.1363 & 0.5043 & 0.1304 & 0.4911 & 0.1352 & 0.5129 & 0.1284 \\
\hline & D & 0.6614 & 0.1230 & 0.6735 & 0.1177 & 0.6659 & 0.1257 & 0.7292 & 0.0779 \\
\hline
\end{tabular}

Table 1(b): Elapsed/CPU Time (in sec.) of the FCM, T2FCM, RFCM and the RT2FCM algorithms for MR Image Segmentation

\begin{tabular}{|c|c|c|c|c|c|}
\hline \multicolumn{2}{|c|}{ Data Set } & FCM & T2FCM & RFCM & RT2FCM \\
\hline \multirow{4}{*}{ 泀 } & $\mathrm{T} 1$ & 4.376156 & 3.497375 & 981.463136 & 717.643003 \\
\hline & $\mathrm{T} 2$ & 2.869447 & 1.214432 & 518.859749 & 664.772973 \\
\hline & PD & 2.280069 & 3.382233 & 477.678632 & 1008.688707 \\
\hline & D & 1.153338 & 2.665022 & 348.290002 & 765.501366 \\
\hline \multirow{4}{*}{ 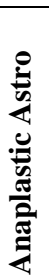 } & T1 & 3.182341 & 2.252212 & 1042.956757 & 596.134572 \\
\hline & $\mathrm{T} 2$ & 4.902215 & 1.286173 & 344.735826 & 512.606467 \\
\hline & PD & 2.397449 & 4.307929 & 892.811375 & 682.858949 \\
\hline & $\mathrm{D}$ & 4.232655 & 4.728278 & 411.677714 & 879.812221 \\
\hline
\end{tabular}




\section{Conclusions}

In this paper we have proposed an enhanced RFCM algorithm where the membership value of each pattern of MR images in RFCM clustering algorithm is extended to the type 2 memberships and is called RT2FCM. From the experimental results it can be seen that the performance of the RT2FCM has better detection of abnormal tissues according to the segmentation results and the values of MSE and IQI as compared to the other existing algorithms for the considered MR images. However, the RT2FCM algorithm has higher computational time as compared to FCM, T2FCM and RFCM clustering algorithm. In future we plan to carry out experimentations with various other patient problems and further improve the computational cost involved with the RT2FCM so that the same can be applied in the real life MR image segmentation and assist medical professionals in making better decisions.

\section{REFERENCES}

[1] Zadeh L. A. 1965. "Fuzzy Sets." Information and control 8: 338-353.

[2] Pawlak Z. 1982. "Rough Sets." International J. Computer and Information Science, vol. 11: 341-356.

[3] Bezdek J.C. 1981. Pattern recognition with fuzzy objective function algorithms. Plenum, New York.

[4] Maji P. and Pal S.K. 2008. "Maximum class separability for Rough-Fuzzy c-means based brain MR image segmentation." LNCS 5390: 114-134.

[5] Maji P. and Pal S.K. 2007. "Rough set based generalized fuzzy c-means algorithm and quantitative indices." IEEE Transaction, on system, man and cybernetics, vol. 37, no. 6, 1529-1540.

[6] Peters G. 2006. "Some refinement of rough k-means clustering." Pattern Recognition 39: 1481-1491.

[7] Zadeh L.A. 1975. "The concept of a linguistic variable and its application to approximate reasoning-1." Informat. Sci, 8: 199-249.

[8] Base A. M. 2004. Pattern Recognition for Medical Imaging. Academic Press, ISBN: 0-12-493290-8.

[9] Tyagi V. and Agarwal J. H. 2009. "Medical Image Processing Overview." Computer Society of India (CSI) Communication, 13-16.
[10] Gonzalez R. C., Woods R. E. and Eddins S. L. 2004. Digital Image Processing Using MATLAB. Pearson Education Inc.

[11] Hall L.O., Bensaid A.M., Clark L. P., Velthuizen R.P. Silbiger M.S. and Bezdek J.C. 1992. "A Comparison of Neural Network and Fuzzy Clustering Techniques in Segmenting Magnetic Resonance Images of the Brain." IEEE Transaction on Neural Networks Vol. 3, No. 5, September: 672-682.

[12] Klir G. J. and Yuan B. 2002. Fuzzy sets and fuzzy logic theory and applications. Prentice Hall of India Private Limited New Delhi.

[13] Yen J. and Langari R. 1999. Fuzzy Logic Intelligence, Control and Information. Pearson Education, Inc.

[14] John R. and Coupland S. 2007. "Type-2 Fuzzy Logic: A Historical View." IEEE Computational Intelligence Magazine February, 1556-603X/07/\$25.00@2007IEEE: 57-62.

[15] Mendel J. M. 2007 (a). "Type-2 Fuzzy Sets and Systems: An Overview." IEEE Computational Intelligence Magazine February, 1556603X/07/\$25.00@2007IEEE: 20-29.

[16] Mendel J. M. 2007 (b). “Advances in type-2 fuzzy sets and systems." Information Sciences 177: 84-110

[17] Kaur P., Lamba I.M.S. and Gosain A. 2011. "A Novel Kernelized Type-2 Fuzzy c-Means Clustering Algorithm in Segmentation of Noisy Brain Tissues." IJSTM, Vol. 2 Issue 3, ISSN: 2229-6646, July: 75-84.

[18] Chaira T. 2011. "A novel intuitionistic fuzzy c- means clustering algorithm and its application to medical images." Applied Soft Computing 11: 1711-1717.

[19] MR image data set, January, .2012. URL: http://myweb.msoe.edu/ martynsc/images/mri/mri.html.

[20] Wang Z. and Bovik A. C. 2009. "Mean squared error: love it or leave it? A new look at signal fidelity measures." IEEE signal processing magazine, January, 1053-5888/09/\$25.00@2009IEEE: 98-117.

[21] Wang Z. and Bovik A. C. 2002. "A universal image quality index." IEEE signal processing letters, vol. 9, no. 3, March: 81-84. 